Results: We studied 84 patients with RA who did not meet FM criteria vs 110 patients who met FM criteria - no differences were seen between patients with primary or secondary FM who met criteria. Patients who met FM criteria were more likely to report each of the 60 symptoms. The 10 symptoms that were most discriminatory of RA vs FM are illustrated in the table 1. A ROC curve for the 60 symptoms vs the FM Criteria had an area of 0.896 (Confidence interval 0.8720.925 ), and for the 10 most discriminatory symptoms of 0.8975 (Confidence interval 0.8667-0.928)

Conclusions: A simple MDHAQ symptom checklist may be informative in routine rheumatology care to identify possible primary and secondary FM in agreement with FM criteria. The MDHAQ may provide a useful and feasible alternative to FM Criteria for busy clinical settings.

Disclosure of Interest: T. Pincus Shareholder of: Dr. Pincus holds a copyright and trademark on MDHAQ and RAPID3 for which he receives royalties and license fees. All revenue is used to support further development of quantitative questionnaire measures for patients and doctors in clinical rheumatology care., S. Jamal: None declared, I. Castrejon: None declared

DOI: 10.1136/annrheumdis-2018-eular.7524

\section{THU0670 A REUMATOLOGIST'S EVALUATION OF HOW EFFICIENCIES IN THE MANAGEMENT OF PAINFUL LUMBAR SYNDROME IN WORK DISABILITY CREATES BENEFITS \& SAVINGS}

V.M. Flores Rodríguez ${ }^{1}$, F. Díaz Gonzalez ${ }^{1}$, S. Bustabad Reyes ${ }^{2}$, M. Carballo Correa ${ }^{3} .{ }^{1}$ Departament of Rheumatology, ${ }^{2}$ Departamento of Rheumatology, University Hospital of the Canary Islands; ${ }^{3} \mathrm{PhD}$ student of the Pharmacy Faculty, University of La Laguna, San Cristóbal de La Laguna, Spain

Background: Musculoskeletal disorders are the most important cause of sick leave in the world. ${ }^{1}$ Lumbar pain is the main cause of temporary incapacity whith significant socio-economic impact.

Objectives: Test efficiency of Healthcare approach by rheumatologist as savings in indirect costs e.g days off work and in direct costs derived from medical assistance in controlled patient group on sick leave diagnosed with lumbar pain.

Methods: 2 year quasi-experimental bi-directional analytical design trial. Retrospective cohort in Control Group- CG and prospective cohort for intervention group -IG. Two groups, IG and CG respectively contain 150 (56\% women aged 47.5 years \pm 10$)$ and $172(48.8 \%$ women aged 44.2 years \pm 10$)$ working age patients with lumbar pain. Study made by Program for the Management of Temporary Disability implemented by Rheumatology Service of University Hospital of the Canary Islands. Study included early intervention, protocol in diagnostic tests and treatment. This study was evaluated by the Ethics Committee of the research of the University Hospital of Canary Islands fulfilling the requirements of suitability. Results: $24 \%$ of patients of IG fit to return to work after first appointment. Maximum efficiency of study between 30 thand 45 th day of sick leave. At 45 days $8 \%$ IG and 18,6\% CG remained on sick leave. Average reduction 26 days of sick leave respect to $C G$, a total saving of 6.182 days of sick leave over 1 year, with efficiency of $62,2 \%$. Saving in direct costs in IG over CG: medicaments, complementary tests, inter-consultations, follow up $48 \%$. Absolute saving of $38.891 €$, or equivalent $256.680 €$ for 1.000 patients. National Institute of Social Security Indirect sick leave costs $67,1 \%$. Absolute saving of $129.883 €$, or equivalent of $857.227 €$ for 1.000 patients. Indirect costs savings for Employers for intervention $50 \%$. Absolute saving $108.649 €$, or equivalent to $717.083 €$ for 1.000 patients. Workers in program saved $50 \%$ in salary loss. Average saving $605 € /$ worker. Total saved by implementation of program to State, to worker, to Employer$360.142 €$. Program Cost effective, producing average annual saving per patient of $259 €$ and average reduction of sick leave days of 41,2 . Program cost efficiencies produced. Every day of disability saved equivalent to implementation cost of $5,06 €$. Cost-benefit program produced for State saving of $6,44 €$ for every $€$ invested in implementation $137.248 €$. Program had $93,8 \%$ patient acceptance expressing maximum satisfaction.

Conclusions: Management of disability by rheumatologist highly profitable given low program implementation cost compared to savings generated for State and Employer.

\section{REFERENCE:}

[1] WHO.The burden of musculoskeletal conditions at the star of the millenium. En: WHO Technical Report Series: 919.Geneva 2003.

Disclosure of Interest: None declared

DOI: 10.1136/annrheumdis-2018-eular.3573
THURSDAY, 14 JUNE 2018

\section{Epidemiology, risk factors for disease or disease progression}

THU0671

STANDARDISED MORTALITY RATES FOR SYSTEMIC LUPUS ERYTHEMATOSUS IN WESTERN AUSTRALIA FROM 1980 TO 2015

W.D. Raymond ${ }^{1}$, D. Preen ${ }^{2}$, H. Keen ${ }^{1}$, C. Inderjeeth ${ }^{1}$, J. Nossent ${ }^{1} .{ }^{1}$ School of Medicine and Pharmacology, ${ }^{2}$ School of Population and Global Health, The University of Western Australia, Perth, Australia

Background: Patients with Systemic Lupus Erythematosus (SLE) are at increased risk of premature mortality. However, population-level mortality rates for SLE have not yet been reported in Australia.

Objectives: Calculate the standardised mortality rates (SMR) for SLE patients in Western Australia (WA) from 1980 to 2015, overall and by age and gender.

Methods: Utilising whole-population linked hospital admission, cancer registration and death data for WA from 1980 to 2015, we compared characteristics and calculated SMRs $(95 \%$ CI) for patients with SLE (ICD-9-CM 695.4 710.0, ICD-10 AM L93.0, M32.0) against controls (5:1) free of rheumatic disease after matching for age, gender, Aboriginality and year of first SLE event.

Results: SLE patients $(n=2,868)$ and controls $(n=12,785)$ recorded 1335 and 4400 deaths with crude mortality rates of $56.3 / 1,000$ vs $37.1 / 1,000$ person-years respectively. SLE patients were approximately 10 years younger (71 vs 81 years) and 2.2-times more likely hospitalised at death $(\mathrm{p}<0.001)$. The age-adjusted SMR (per 1,000) for SLE patients was $4.4(95 \% \mathrm{Cl} 3.0,5.8)$, and higher in females 5.4 $(95 \% \mathrm{Cl} 3.5,7.4)$ than in males $3.4(95 \% \mathrm{Cl} 1.5,5.4)$. Five-year period SMRs were $8.6(95 \% \mathrm{Cl} 5.0,12.2)$ between $1990-1994,9.3(95 \% \mathrm{Cl} 4.4,14.2)$ between 1995 $1999,7.6(95 \% \mathrm{Cl} 3.1,12.1)$ between $2000-2004,4.5(95 \% \mathrm{Cl} 0.2,8.7)$ between 2005-2009, and 4.9 (95\%Cl 1.32, 8.5) between 2010-2015.

Conclusions: SLE patients in WA experienced a decline in SMRs over time, but remain at increased risk of premature mortality. Within the limitations of administrative linked data, SLE in WA associates with an average reduction in life span of 10 years.

Acknowledgements: The authors wish to thank the staff at the Western Australian Data Linkage Branch and Emergency Department Data Collection, Hospital Morbidity Data Collection, WA Cancer Registry and Death Registrations.

The Rheumatology Group of UWA (JN) was supported by an unrestricted grant from the Arthritis and Osteoporosis Foundation of Western Australia (AOWA). The AOWA provided WR with a PhD Scholarship in Memory of Johan Donald Stewart. This research was also supported by an unrestricted Australian Project Grant from Arthritis Australia.

Disclosure of Interest: None declared

DOI: 10.1136/annrheumdis-2018-eular.6142

\section{THU0672 IMPACT OF PREGNANCY ON PHYSICAL FUNCTION AND HEALTH-RELATED QUALITY OF LIFE IN WOMEN WITH AXIAL SPONDYLOARTHRITIS}

K. Ursin ${ }^{1}$, S. Lydersen ${ }^{2}$, J. Skomsvoll ${ }^{1}$, M. Wallenius ${ }^{1} .{ }^{1}$ National advisory unit on pregnancy and rheumatic diseases, Department of rheumatology, St. Olavs hospital; ${ }^{2}$ Regional center for child and youth mental health and child welfare, Faculty of Medicine and health sciences, Norwegian University of science and technology, Trondheim, Norway

Background: The only previous study exploring the impact of pregnancy on health-related quality of life in women with axial spondyloarthritis (axSpA), included only ten women. ${ }^{1}$

Objectives: To prospectively study physical function and health-related quality of life in a large cohort of women with axSpA during and after pregnancy, using Bath Ankylosing Spondylitis Functional Index (BASFI) and three of the dimensions of RAND 36-Item Health Survey (RAND-36): Physical functioning, bodily pain and mental health.

Methods: RevNatus is a Norwegian nationwide register designed for the followup of pregnant women with rheumatic diseases. Our study comprised 179 pregnancies in 166 women with axSpA included in RevNatus between 2006 - 2016 The women had seven visits at a rheumatology unit; before pregnancy, in each trimester, and six weeks, six months and twelve months postpartum. BASFI-scores and scores of RAND-36 physical functioning, bodily pain and mental health from each visit were analysed in a linear mixed model. BASFI has an overall index score between 0 ("no functional impairment") and 10 ("maximal functional 
impairment"). ${ }^{2}$ The dimensions of RAND-36 have scores between 0-100 (where $100=$ "best possible health"). ${ }^{3}$

Results: BASFI-scores were highest in third trimester, significantly higher than six weeks postpartum (mean BASFI 3.6 vs. 2.6, p<0.001). In line with this result, scores of RAND-36 physical functioning was significantly lower in third trimester compared to six weeks postpartum (mean physical function 54.5 vs. 71.0, $\mathrm{p}<0.001$ ). The women reported considerable pain throughout the study period, with worst reported pain in second trimester, when scores of RAND-36 bodily pain were significantly lower than six weeks after postpartum (mean bodily pain 44.3 vs. 49.6, $\mathrm{p}=0.01$ ). Scores of RAND-36 mental health were high and stable throughout the study period, with best reported mental health six weeks after delivery, when mental health was significantly better than in all time points in pregnancy.
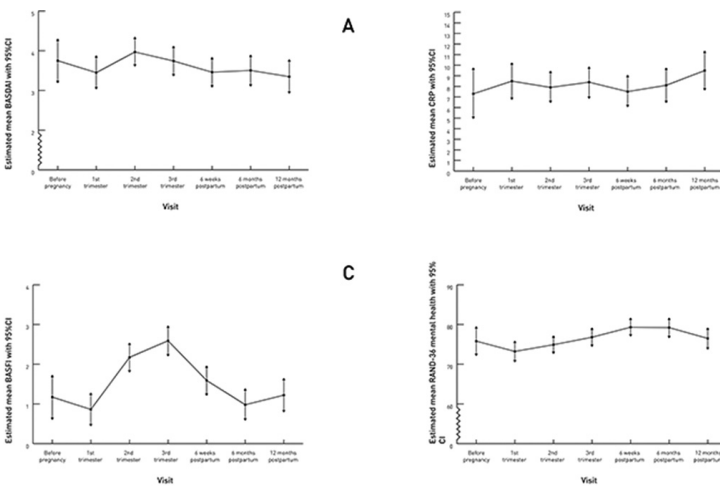

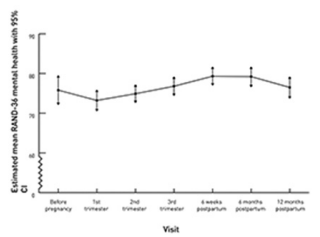

D

Abstract THU0672 - figure 1. shows changes in functionality and mental health from preconception to one year after delivery, and disease activity and CRP in the same time period.

Conclusions: Even though women with axSpA reported considerable pain and gradually worsening of physical function in pregnancy, they experienced good and stable mental health from preconception to one year after delivery.

\section{REFERENCES:}

[1] Forger F, Ostensen M, Schumacher A, Villiger PM. Impact of pregnancy on health related quality of life evaluated prospectively in pregnant women with rheumatic diseases by the SF-36 health survey. Ann Rheum Dis 2005;64(10):1494-9.

[2] Sieper J, Rudwaleit M, Baraliakos X, Brandt J, Braun J, Burgos-Vargas R, et al. The Assessment of SpondyloArthritis international Society (ASAS) handbook: a guide to assess spondyloarthritis. Ann Rheum Dis 2009;68 (Suppl 2):ii1-44.

[3] Hays RD, Sherbourne CD, Mazel RM. The RAND 36-Item Health Survey 1.0. Health Econ 1993;2(3):217-27.

Disclosure of Interest: None declared

DOI: 10.1136/annrheumdis-2018-eular.1603

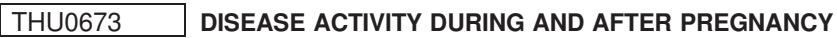 IN WOMEN WITH PSORIATIC ARTHRITIS}

K. Ursin ${ }^{1}$, S. Lydersen ${ }^{2}$, J. Skomsvoll ${ }^{1}$, M. Wallenius ${ }^{1} .{ }^{1}$ National advisory unit on pregnancy and rheumatic diseases, Department of rheumatology, St. Olavs hospital; ${ }^{2}$ Regional center for child and youth mental health and child welfare, Faculty of Medicine and health sciences, Norwegian university of science and technology, Trondheim, Norway

Background: The few previous studies on disease activity of psoriatic arthritis in pregnancy have shown diverging results. ${ }^{1-3}$ None of the studies used validated disease activity measures and the only prospective study was conducted before the widespread use of biological DMARDs.

Objectives: The aim of this project was to prospectively study disease activity in women with psoriatic arthritis before, during and after pregnancy with DAS28CRP-3 as disease activity measure.

Methods: RevNatus is a Norwegian nationwide register designed for the followup of pregnant women with rheumatic diseases. Our study comprised 108 pregnancies in 103 women with psoriatic arthritis with mainly peripheral involvement, included in RevNatus between 2006 - 2017. The women had seven visits at a rheumatology unit; before pregnancy, in each trimester, and six weeks, six months and twelve months postpartum. DAS28-CRP-3-scores from each visit were analysed in a linear mixed model. We did additional analyses with "tumour necrosis factor inhibitor (TNFi) in pregnancy (yes/no)" as covariate.

Results: Altogether, our study demonstrated stable, low disease activity during and after pregnancy in women with psoriatic arthritis. However, as shown in figure 1 , disease activity decreased in pregnancy and increased within six months postpartum, with disease activity six months postpartum significantly higher than six weeks postpartum (estimated mean DAS28 2.71 vs. 2.45, $\mathrm{p}=0.016$ ). Women using TNFi in pregnancy had significantly lower disease activity than women not using TNFi (estimated mean DAS28 six weeks postpartum 1.97 vs. 2.48, $\mathrm{p}=0.039$ ).
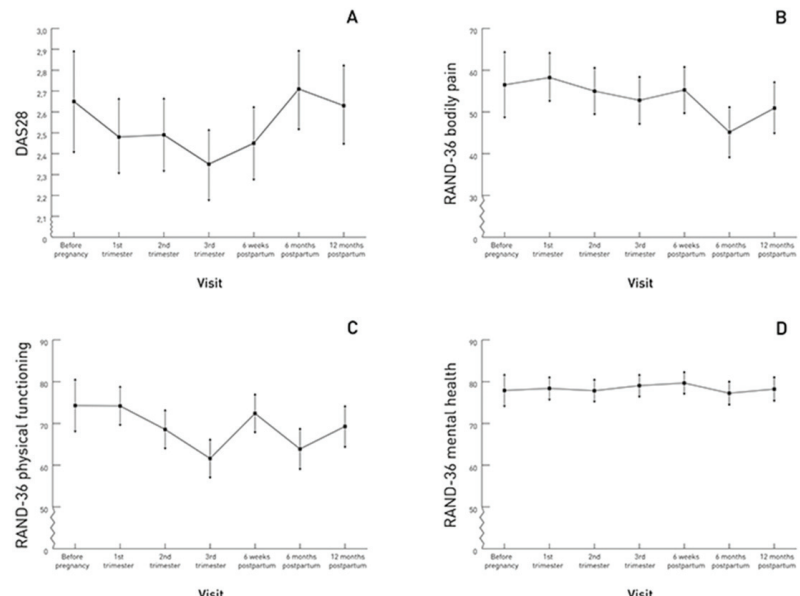

Abstract THU0673 - Figure 1. also shows self-reported pain, physical functioning and mental health throughout the study period.

Conclusions: Studying women with psoriatic arthritis, we found that disease activity was highest six months postpartum, but altogether stable in the period from planning pregnancy to one year after delivery. Women using TNFi in pregnancy had significantly lower disease activity.

\section{REFERENCES}

[1] Ostensen M. The effect of pregnancy on ankylosing spondylitis, psoriatic arthritis, and juvenile rheumatoid arthritis. Am J Reprod Immunol 1992;28 (3-4):235-7.

[2] Mouyis MA, Thornton CC, Williams D, Giles IP. Pregnancy Outcomes in Patients with Psoriatic Arthritis. J Rheumatol 2017:44(1):128-9.

[3] Polachek A, Li S, Polachek IS, Chandran V, Gladman D. Psoriatic arthritis disease activity during pregnancy and the first-year postpartum. Semin Arthritis Rheum 2017.

Disclosure of Interest: None declared

DOI: 10.1136/annrheumdis-2018-eular.1604

\section{THU0674 RHEUMATIC DISEASES AND PREGNANCY: A SINGLE} CENTRE DEDICATED CLINIC EXPERIENCE

F. Motta ${ }^{1,2}$, V. Ramoni ${ }^{1}$, B. Vitolo ${ }^{1}$, A. Milanesi $^{1}$, F. Beneventi $^{3}$, S. Quaglini ${ }^{4}$, R. Caporali ${ }^{1,2}$, C. Montecucco ${ }^{1,2}$. ${ }^{1}$ Unit of Rheumatology, IRCCS Foundation Policlinico San Matteo; ${ }^{2}$ University of Pavia; ${ }^{3}$ Department of Obstetrics and Gynecology, IRCCS Foundation Policlinico San Matteo and University of Pavia; ${ }^{4}$ Department of Electrical, Computer and Biomedical Engineering, University of Pavia, Pavia, Italy

Background: Pregnancy causes immune and endocrine systems alterations therefore it can change the course of the rheumatic diseases. On the other hand rheumatic diseases can negatively influence pregnancy outcome. Therefore, in the past pregnancy has been discouraged in patients with rheumatic diseases, for high maternal-fetal risks. Nowadays we are aware that counselling, planning of pregnancy, multidisciplinary management and follow up in a dedicated clinic are mandatory for a good maternal-fetal outcome.

Objectives: To assess the impact of close multidisciplinay rheumatological obstetrical management on high risk pregnancies.

Methods: We considered patients diagnosed with a rheumatic disease before pregnancy who had at least one pregnancy prospectively followed at our dedicated clinic. Each patient underwent a monthly rheumatological and obstetrical evaluation during the all pregnancy. All obstetrical complications were recorded and a final pregnancy outcome was assessed: favourable outcome (delivery) or 